\title{
Preparation of polyols from methyl- $\alpha$-D-glucoside and cyclic esters for design and fabrication of biodegradable polyurethane foams
}

\begin{abstract}
A ring-opening polymerization of cyclic esters $\varepsilon$-caprolactone $(\mathrm{CL})$ and/or L-lactide (LACD) was carried out in the presence of methyl- $\alpha$-D-glucoside (m-Glc) as an activated substrate and tin(II) 2-ethylhexanoate $\left(\mathrm{SnEht}_{2}\right)$ as a catalyst. This led to successful synthesis of an armed polyester polyol with $\mathrm{m}$-Glc as the core. These polyols were characterized through gel permeation chromatography, hydroxyl value assessment, differential scanning calorimetry, and viscosity measurements. It was clarified that the thermal properties and tractability of the synthesized polyols are closely related to the structural characteristics of the side chain polyesters, such as their length and chemical composition. Polyurethane foams were also prepared by mixing an appropriate amount of the polyols with diphenyl methane diisocyanate, a foaming agent (water), and other additives, and their mechanical properties were examined. Two polyurethane foams manufactured from polycaprolactone-polyols that were synthesized at a CL/mGlc ratio of 2 or 5 , were used in a biodegradation test in a closed system of activated sludge suspension. Evidence of their biodegradation is presented based on data showing oxygen consumption by the pulverized samples.
\end{abstract}

Key words Methyl- $\alpha$-D-glucoside $\cdot \varepsilon$-Caprolactone $\cdot$ LLactide $\cdot$ Polyurethane foam $\cdot$ Biodegradability

M. Yoshioka $(\bowtie) \cdot$ A. Miyata $\cdot$ Y. Nishio

Division of Forest and Biomaterials Science, Graduate School of Agriculture, Kyoto University, Kyoto 606-8502, Japan

Tel. +81-75-753-6253; Fax +81-75-753-6300

e-mail: mariko@kais.kyoto-u.ac.jp

T. Yagi

Toxicological and Environmental Sciences Center, Mitsui Chemical Analysis and Consulting Service Inc., Chiba 297-0017, Japan

\section{Introduction}

A long time has passed since the invention of polyurethanes by Bayer in 1937 and since than those polymers have found applications in a wide range of products. One of the most important commercial polyurethane products is foam. Polyurethanes are copolymers containing blocks of relatively low molecular weight polyesters or polyethers that are covalently bonded by a urethane group ( $-\mathrm{NHCO}-\mathrm{O}-$ ). The polymers are synthesized via reaction of two basic components, polyisocyanate and polyhydroxyl-containing polymer (i.e., polyester or polyether polyols). Although the majority of current polyols used for polyurethane manufacture are derived from petrochemical products, there is pressing need to find an alternative source of polyols for polyurethane syntheses. A promising source is that of naturally occurring and renewable plants. Among plant-origin substances, the simplest and most abundantly usable material is glucose. However, very few trials have been carried out for converting glucose into polyols for polyurethane. Even in the known cases, solvents were used in the process, in which glucose was dissolved at a very low concentration of $2 \%-8 \%$, and many of by-products were formed during the conversion. ${ }^{1,2}$

The present study is concerned with new structural design and synthesis of polyols to develop biodegradable polyurethane foams by utilizing plant biomass. In our preliminary work, ${ }^{3,4} \mathrm{D}$-glucose was used as a starting material to prepare polyols for polyurethanes, which should be liquid at room temperature. This was conducted by polymerization of $\varepsilon$-caprolactone $(\mathrm{CL})$ with initiation at the hydroxyl groups of glucose and employed tin(II) 2ethylhexanoate $\left(\mathrm{SnEht}_{2}\right)$ as the catalyst. In this case, because of the existence of the unstable cyclic hemiacetal anomers of glucose, dehydration, oxidation, and pyrolysis reactions occurred under the polyol synthesis conditions, i.e., at $150^{\circ} \mathrm{C}$. Thus, the polyols obtained contained various by-products, the chemical structures of which were unknown, that were dark brown in color. In order to obtain polyols with high biodegradability and high safety of the 
intermediate formed during biodegradation, the destruction of the glucose structure is fundamentally undesirable and should be avoided.

For the above reason, methyl $\alpha$-D-glucoside (m-Glc) was selected as the hydroxyl-containing substrate, in which the anomeric carbon of glucose is blocked by a methyl substituent to give a stable acetal structure. As a result of ringopening polymerization of $\mathrm{CL}$ on the m-Glc hydroxyls, polyester polyols were prepared without significant destruction of the pyranose ring and without color development. The products assumed a liquid state at room temperature only when the in-feed ratio [liquid ratio (LR)] of the monomer to $\mathrm{m}-\mathrm{Glc}$ is less than 3 . When LR became larger than 3, the introduced polycaprolactone (PCL) side chains were liable to aggregate with each other. This trend was enhanced with an increase in the LR value, resulting in formation of the crystal structure below the melting temperature of PCL. One of the requirements for polyols to produce polyurethane is that they should be liquid with a processible viscosity at room temperature. In this sense, weakening of the ordered interaction among the introduced side chains became an objective of this study. In the present report, results of the investigation trying to reduce, as much as possible, the interaction among the polyester side chains is described.

\section{Experimental}

\section{Materials}

Chemicals for polyol synthesis included m-Glc (Nacalai Tesque), CL (Placcel M, Daicel Chemical), SnEht ${ }_{2}$ (Wako), and L-lactide (LACD) (Purac, Japan). Solvents for measurements of molecular weights, their distribution, and hydroxyl values, and other reagents were purchased from Nacalai Tesque and Wako.

Chemicals for preparing polyurethane foams included polymeric diphenyl methane diisocyanate (MDI) (Nippon Polyurethane), dibutyl-tin-dilaurate as a catalyst, a surfactant SH-193 (Dow Corning Toray Silicone), and distilled water as a foaming agent.

Among the above reagents, CL monomer was vacuumdistilled before use, but others were used as received.

Synthesis of polyols

Air-dried m-Glc was weighed into a four-neck flask, vacuum-dried for more than $12 \mathrm{~h}$ at $60^{\circ} \mathrm{C}$, and weighed to evaluate the absolute dry weight. Two to 20 times excess amounts of CL and/or LACD relative to the weight of $\mathrm{m}$ Glc were added to the flask and a stirrer was fitted with it. Stirring was continued at $150^{\circ} \mathrm{C}$ for 30 min under a nitrogen atmosphere. When solid powdered LACD existed in the flask, special care was taken; that is, vigorous stirring was only applied after complete dissolution of LACD. An amount of $\mathrm{SnEht}_{2}$, that was $0.33 \%$ with respect to the total

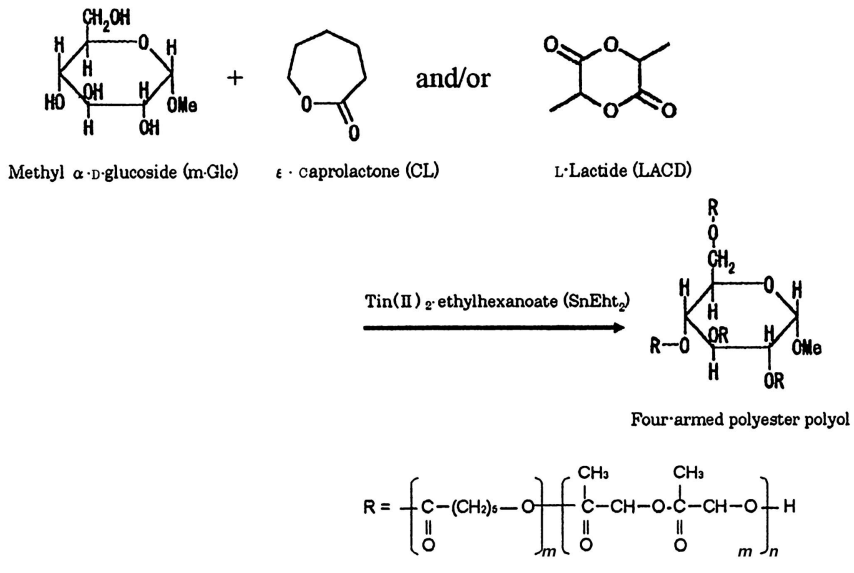

Fig. 1. Synthesis of four-armed polyester polyol from methyl- $\alpha$-Dglucoside (m-Glc) and $\varepsilon$-caprolactone (CL) and/or L-lactide (LACD)

weight of the components in the flask, was added and the reaction was continued for $150 \mathrm{~min}$ (Fig. 1).

Gel permeation chromatography analysis

Gel permeation chromatography (GPC) analysis was carried out for polyol products. The molecular weight distribution of the respective samples was determined on a Tosoh HLC-8020 gel permeation chromatograph equipped with a refractive index (RI) detector and using two columns (TSK-GEL $\mathrm{G} 2000 \mathrm{H}_{\mathrm{HR}}$ and $\mathrm{G}_{1000 \mathrm{H}_{\mathrm{HR}}}$ ) connected in series. The measurement was conducted by using tetrahydrofuran (THF) as the mobile phase at a flow rate of $1.0 \mathrm{ml} / \mathrm{min}$. The concentration of the test samples was $0.5 \%(\mathrm{w} / \mathrm{v})$ in THF and the injection volume was $100 \mu$ l. The system was calibrated with monodisperse polystyrene standards.

Hydroxyl value determination

The hydroxyl numbers of polyols were measured ${ }^{5}$ to determine the amount of diisocyanate required for the preparation of foams from the polyols. Polyol $(1.0 \mathrm{~g})$ was accurately weighed into a $100-\mathrm{ml}$ beaker and a phthalating agent $(25 \mathrm{ml})$ was added. The phthalating agent was composed of a mixture of phthalic acid anhydride $(15 \mathrm{~g})$, dioxane $(100 \mathrm{ml})$, and imidazole $(2.42 \mathrm{~g})$. The beaker was heated on a hot plate $\left(110^{\circ}-130^{\circ} \mathrm{C}\right)$ and maintained for $20 \mathrm{~min}$ under boiling to complete the phthalating reaction of hydroxyl groups. After cooling to room temperature, $50 \mathrm{ml}$ of dioxane and $25 \mathrm{ml}$ of deionized water were added into the beaker and stirred thoroughly. After the contents were completely dissolved, the remaining carboxyl groups were titrated with $1 \mathrm{~N}$ sodium hydroxide solution using a potentiometric titration apparatus AT-510 (Kyoto Electronics). The hydroxyl value was calculated according to the following equation:

Hydroxyl value $(\mathrm{mg} \mathrm{KOH} / \mathrm{g})=[(B-A) \times f \times 56.1] / W$ 
where $A$ is the titer for the sample $(\mathrm{ml}), B$ is the blank titer $(\mathrm{ml}), f$ is the titration factor of the aqueous sodium hydroxide solution, and $W$ is the weight of the polyol sample $(\mathrm{g})$.

\section{Differential scanning calorimetry}

Differential scanning calorimetry (DSC) analysis was carried out for polyol samples using a Seiko DSC6200/ EXSTAR6000. The temperature program was made up of three stages: heating from room temperature to $200^{\circ} \mathrm{C}$, quenching from $200^{\circ} \mathrm{C}$ to $-140^{\circ} \mathrm{C}$, and again heating from $-140^{\circ} \mathrm{C}$ to $280^{\circ} \mathrm{C}$, each conducted in a nitrogen atmosphere. The scanning rate was $20^{\circ} \mathrm{C} / \mathrm{min}$ and the sample weight was ca. $10 \mathrm{mg}$. Data obtained in the second heating scan was used for the analysis. For estimation of the glass transition temperature $\left(T_{\mathrm{g}}\right)$ of each sample, the midpoint of a discontinuity in heat flow was noted. The melting temperature $\left(T_{\mathrm{m}}\right)$ was determined from the maximum of the melting endothermic peak.

\section{Viscosity measurement}

The viscosities of the polyols obtained were measured with a rotation viscometer B8H (Toki Sangyo) in a room conditioned at $20^{\circ} \mathrm{C}$ and $60 \%$ relative humidity (RH). The average value of three repeated measurements was taken as the data for each sample.

\section{Foam production process}

The isocyanate index required for making foams was usually around 110. A surfactant (silicone oil, SH-193), a catalyst (n-dibutyl-tin-dilaurate), and water were used at concentrations of $4 \%-6 \%, 0.7 \%-1.2 \%$, and $6 \%$, respectively, based on the weight of a given polyol.

Weighed amounts of the polyol, catalyst, surfactant, and water were first mixed well in a paper cup. Next, an appropriate amount of polymeric MDI was added to the mixture and stirred with a high-speed agitator $(15000 \mathrm{rpm})$ for $15 \mathrm{~s}$. Immediately after the stirring, creaming and foaming took place in the paper vessel. A scheme of the possible reactions leading to the polyurethane foam is shown in Fig. 2. The foams obtained were cured at room temperature for more than $24 \mathrm{~h}$ before measurements were performed.

The isocyanate index was calculated by the following equation:

Isocyanate index $=\left[\left(M_{\mathrm{MDI}} \times W_{\mathrm{MDI}}\right) /\left(M_{\text {polyol }} \times W_{\text {polyol }}+\right.\right.$ $\left.\left.M_{\mathrm{W}} \times W_{\mathrm{w}}\right)\right] \times 100$

where $M_{\mathrm{MDI}}$ is the mole number of isocyanate groups per gram of MDI, $W_{\mathrm{MDI}}$ is the gram weight of MDI, $M_{\text {polyol }}$ and $M_{\mathrm{w}}$ are the mole number of hydroxyl groups per gram of the polyol and of water, respectively, and $W_{\text {polyol }}$ and $W_{\mathrm{w}}$ are the gram weights of the polyol and water, respectively, in the foam preparation.

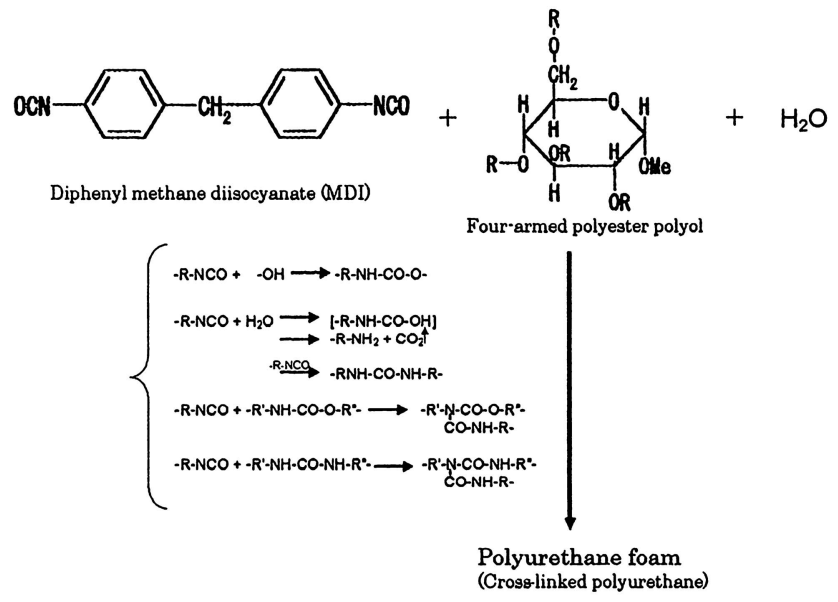

Fig. 2. A possible reaction scheme of the synthesis of polyurethane foam from diphenyl methane diisocyanate (MDI) and four-armed polyester polyol

\section{Compression strength measurements}

Compression strength of foams was measured based on the JIS K7220 standard. The foams tested were cut into specimens measuring $50 \times 50 \times 50 \mathrm{~mm}$. The specimens were conditioned at $20^{\circ} \mathrm{C}$ and $60 \% \mathrm{RH}$ for $48 \mathrm{~h}$. The measurement was conducted with a Shimadzu Autograph AGS$5 \mathrm{kNG}$ (Shimadzu) in the directions perpendicular and parallel to the foam rise. A load was applied at a crosshead speed of $5 \mathrm{~mm} / \mathrm{min}$ until the specimen was compressed to approximately $10 \%$ of its original thickness.

Measurements of oxygen consumption in activated sludge

According to the procedure OECD guideline $302 \mathrm{C}$ for testing of chemicals, an activated sludge adjusted to $\mathrm{pH} 7.0$ was prepared by adding finely cryogenically pulverized foam $(30 \mathrm{ppmw} / \mathrm{v})$ and standard activated sludge (100 ppm w/v) into a basic culture solution. The biochemical oxygen demand (BOD) for the powdered sample was measured at $25^{\circ} \mathrm{C}$ over a period of 104 days. The amount of theoretical oxygen consumption for the complete mineralization was calculated from elemental analysis data for each sample. The biodegradability was evaluated by a specific value, the degree of biodegradation (\%), which can be obtained as the proportion of the actual oxygen consumption to the theoretical consumption.

\section{Results and discussion}

Synthesis of polyols

$m-G l c / C L$ reaction system

When m-Glc was added to CL at a LR (weight ratio of CL to $\mathrm{m}-\mathrm{Glc}$ ) of more than 2 , the former easily dissolved in the 
latter with stirring at $150^{\circ} \mathrm{C}$. Even in the case where LR is 2, the dissolution was completed when the catalyst $\left(\mathrm{SnEht}_{2}\right)$ was added and stirred at $150^{\circ} \mathrm{C}$. All the solutions were, more or less, amber in color. With the advance of the reaction, however, the mixtures having large LR values became turbid and white in color. The higher the LR, the more enhanced the cloudiness. When the reaction mixtures were cooled to room temperature after the prescribed reaction time, the products obtained with LR less than 3 were transparent amber liquids, whereas those with LR of 3 or higher became clouded and usually did not flow at room temperature. When the LR exceeded 5, the coagulation property was pronounced.

The molecular weight determination was carried out by GPC for the product materials. Three GPC curves are shown in Fig. 3 for polyols synthesized at LRs of 2, 5, and 20. The use of reaction conditions of $150^{\circ} \mathrm{C} / 150 \mathrm{~min}$ led to the disappearance of the monomer peak at a retention time

(a)

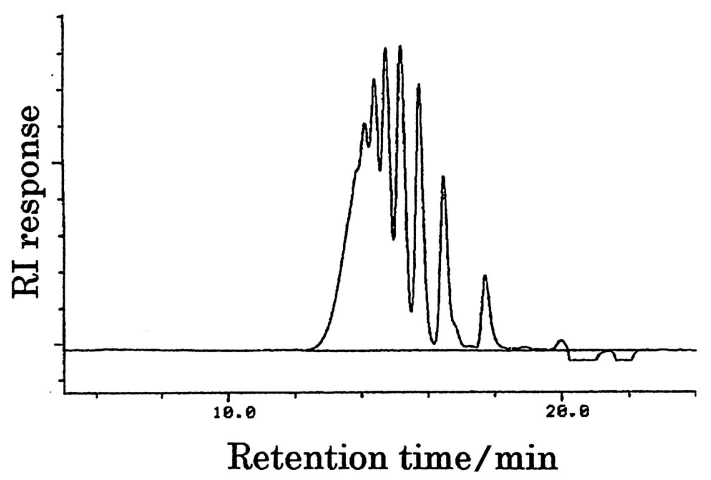

(b)

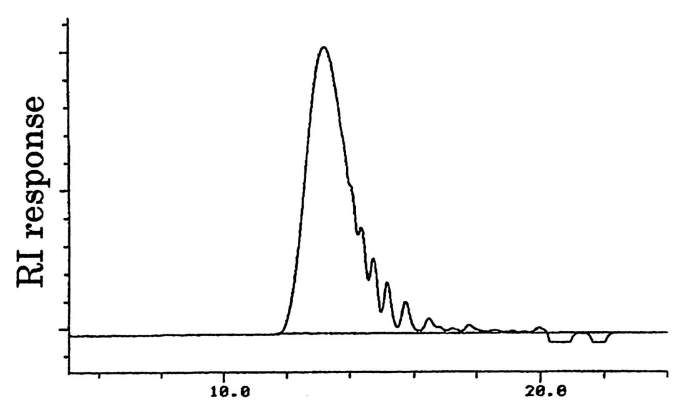

Retention time/min

(c)

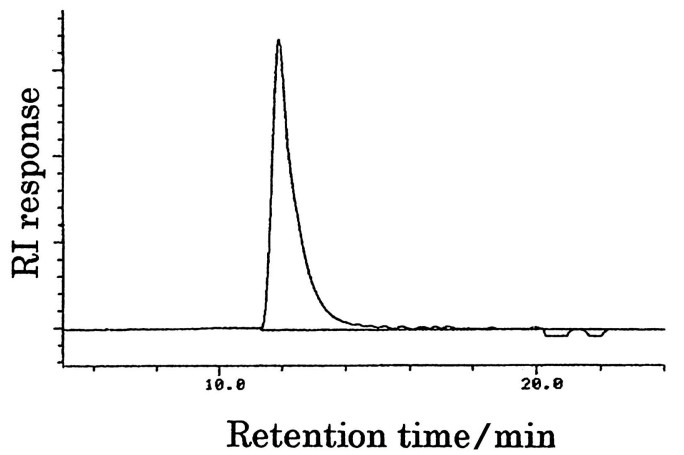

Fig. 3a-c. Gel permeation chromatography (GPC) chromatograms of polyols synthesized under reaction conditions of $150^{\circ} \mathrm{C} / 150 \mathrm{~min}$. (a) $\mathrm{m}-\mathrm{Glc} / \mathrm{CL}=1 / 2(\mathrm{LR}=2),(\mathbf{b}) \mathrm{m}-\mathrm{Glc} / \mathrm{CL}=1 / 5(\mathrm{LR}=5)$, (c) $\mathrm{m}-\mathrm{Glc} / \mathrm{CL}=1 / 20(\mathrm{LR}=20)$ (by weight)
(RT) of $19.97 \mathrm{~min}$, indicating that the reaction proceeded well. From a literature report ${ }^{6}$ and our previous data, ${ }^{7}$ it may be pointed out that only the ring-opening polymerization initiated at the hydroxyl positions in m-Glc progresses and no homopolymer is produced if water is excluded from the reaction system.

As can be seen from the GPC data in Fig. 3, the molecular weight of the product material becomes higher with the increase in the LR value. In Fig. 4, values of the weight average molecular weight obtained from the GPC analysis are plotted against LR. From the plot, it is found that the molecular weight increases almost linearly with increasing LR.

For the purpose of synthesizing polyols for polyurethane, it is desirable that they are liquid at room temperature. Accordingly, it can be said from the above observation that the polymerization of CL should be performed with LRs less than 3. The adoption of such small LR values, however, will cause other problems, e.g., difficulty in attaining adequate hydroxyl numbers required for urethane-resin foaming, undesirable physical properties of the product foams, and so forth. Thus, further studies for preparing improved polyols from $\mathrm{m}$-Glc are required.

\section{$m-G l c / L A C D$ reaction system}

The synthesis of polyols from m-Glc and LACD was carried out with LRs of 2 and 5 as representative cases for using a small LR and a large one, respectively. Before addition of catalyst, m-Glc partly dissolved in LACD with heating at $150^{\circ} \mathrm{C}$. After the addition, the m-Glc dissolved completely at the same temperature with gradual development of an amber color. In both cases (LR 2 and 5), the viscosity increased remarkably during polymerization of LACD and the products were amber-colored solids at room temperature. The products still showed high viscosity at $60^{\circ} \mathrm{C}$, and behaved like a glutinous starch syrup. Thus, the products were unusable as polyols for polyurethane foam preparation. As a conclusion, it can be said that the production of oligoesters derived from LACD and m-Glc develops less

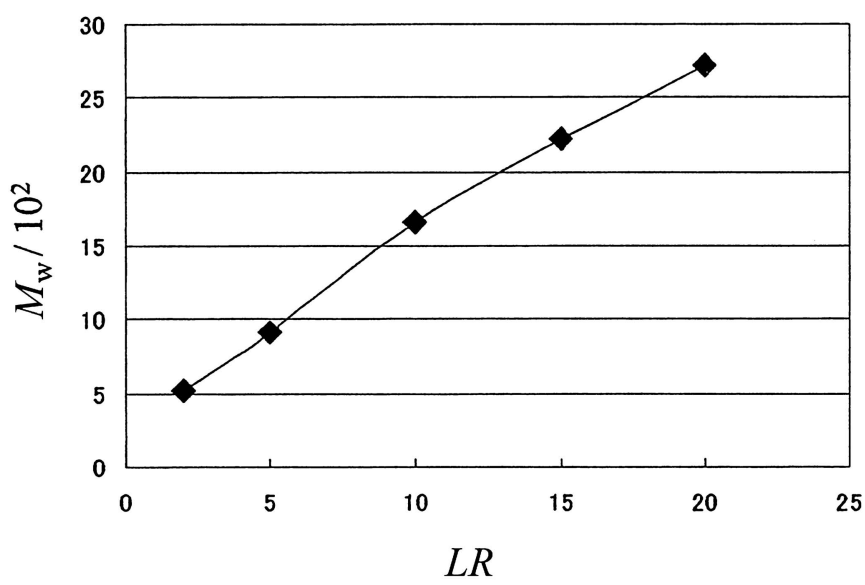

Fig. 4. Weight average molecular weights obtained by GPC, plotted against LR (weight ratio of CL to $\mathrm{m}-\mathrm{Glc}$ ) 
plasticization compared with the introduction of CL side chains.

$$
m-G l c /(C L+L A C D) \text { reaction system }
$$

As has been described above, it is desirable in preparation of objective polyurethanes to synthesize polyols in which oligoester side chains attached to m-Glc are long enough to give appropriate hydroxyl values and, at the same time, to assume a liquid state at room temperature. In this sense, the two examples of polyol synthesis mentioned above were not always successful.

An attempt was made to introduce cooligoester side chains by the combined use of LACD and CL. It is expected that a interaction or self-assembly of the side chains is minimal due to irregularity in their arrangement of repeating units. Thus, such polyols were synthesized from m-Glc in the presence of both CL and $\mathrm{LACD}$ at $150^{\circ} \mathrm{C}$. Molar ratios of CL:LACD were 5:5, 7:3, 8:2, and 9:1 and LRs of 2, 5, 10 , and 15 were adopted, where LR denotes the weight ratio of CL + LACD to m-Glc. Amber coloration of the reactant liquids developed with the progress of polymerization reaction. The polyols derived from CL:LACD molar ratios of 7:3 and 8:2 were found to assume the liquid state, even after cooling to room temperature (see Fig. 5).

The viscosities of the synthesized polyols, measured at $20^{\circ} \mathrm{C}$, are listed in Table 1 . The viscosity values were largely dependent on the molar ratio of CL:LACD as well as on the $\mathrm{LR}$ value. The CL:LACD ratio of $8: 2$ and relatively small values of LR gave low viscosities for the polyol products. Taking account of the viscosity values ${ }^{8}$ of commercial polyester polyols for soft polyurethane foams (14000$20000 \mathrm{mPa} \cdot \mathrm{s})$ and those for semisoft polyurethane foams $(18000-24000 \mathrm{mPa} \cdot \mathrm{s})$, the viscosity data shown in Table 1

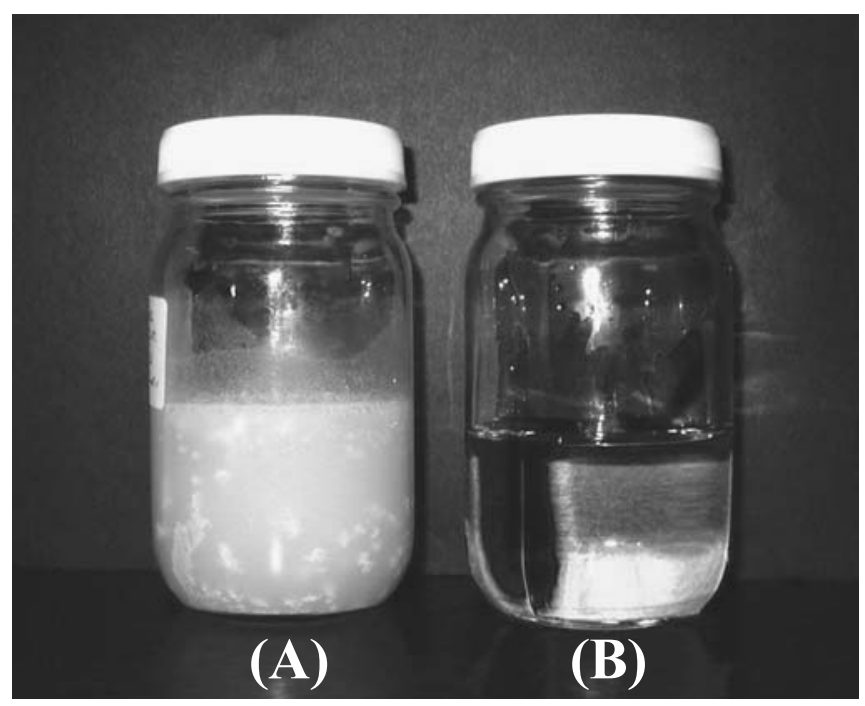

Fig. 5. Visual appearance of polyols synthesized under conditions of $150^{\circ} \mathrm{C} / 150 \mathrm{~min}$. In-feed chemical compositions: $\mathbf{A}, \mathrm{m}-\mathrm{Glc} / \mathrm{CL}=1 / 5(\mathrm{LR}$ $=5)($ by weight $) ; \mathbf{B}, \mathrm{m}-\mathrm{Glc} /(\mathrm{CL}+\mathrm{LACD})=1 / 5(\mathrm{LR}=5)$ (by weight $)$ and $\mathrm{CL} / \mathrm{LACD}=8 / 2$ (by mole) may be industrially acceptable. The successful synthesis of liquid polyols showing acceptable viscosity values may be attributed primarily to the structural irregularity of the introduced side chains, which prevents their crystallization. It should also be emphasized that a wide variety of products are obtainable as attractive polyols for polyurethane by differing the chain length and the chemical composition of the introduced oligocopolyesters.

In the syntheses with a CL:LACD molar ratio of 9:1 and when LR became 10 or more, the viscosity and cloudiness of the reaction mixtures increased noticeably. This was considered to be due to the aggregation attended by crystallization among PCL sequences in the introduced side chains. Conversely, in the case of a CL:LACD ratio of 5:5, a comparatively rigid nature of polylactide (PLA) segments was prominent, as observed with the viscosity of the reactant mixture becoming higher with the progress of the polymerization reaction. The polyol products showed poor flow properties at room temperature, although they were situated above $T_{\mathrm{g}}$ (see Fig. 7). The abundance of PLA sequences in the side chains may be explained by a higher rate of the ring-opening polymerization of LACD in this reaction system compared with that of CL. ${ }^{6}$ Thus, it is necessary to ensure that the monomer reactant mixture has molar proportions of $<50 \% \mathrm{LACD}$ and $<90 \% \mathrm{CL}$ in order to avoid the formation of significant lengths of homopolymer structure of LACD or CL leading to some agglomeration among the side chains. Eventually, the polyols synthesized with CL:LACD molar ratios of $8: 2$ and 7:3 for LRs of 515 , gave a reasonable hydroxyl value within an acceptable range of $50-400 \mathrm{mg} \mathrm{KOH} / \mathrm{g}$ polyol $^{7}$, as shown in Table 1 . A monomer ratio of 9:1 was also acceptable when the LR was 5. These materials showed low viscosity at room temperature, and were capable of mixing satisfactorily with other reagents used for foam production. It should also be noted that the above type of polyols with hydroxyl numbers up to about $800 \mathrm{mg} \mathrm{KOH} / \mathrm{g}$ polyol would be utilizable if they can be diluted compatibly with other commercial polyols.

Table 1. Hydroxyl values and viscosities of polyols synthesized

\begin{tabular}{llll}
\hline Composition & & $\begin{array}{l}\text { OH value } \\
(\mathrm{mg} \mathrm{KOH} / \mathrm{g})\end{array}$ & $\begin{array}{l}\text { Viscosity/10 } \\
(\mathrm{mPa} \cdot \mathrm{s})\end{array}$ \\
\cline { 1 - 2 } $\begin{array}{llll}\text { m-Glc/(CL + LACD) } \\
\text { (by weight) }\end{array}$ & $\begin{array}{l}\text { CL/LACD } \\
\text { (by mole) }\end{array}$ & & \\
\hline $1 / 5(\mathrm{LR}=5)$ & $5 / 5$ & 172.5 & $\mathrm{NE}$ \\
& $7 / 3$ & 190.0 & 1.95 \\
& $8 / 2$ & 224.1 & 1.09 \\
& $9 / 1$ & 199.9 & 1.03 \\
$1 / 10(\mathrm{LR}=10)$ & $5 / 5$ & 105.1 & $\mathrm{NE}$ \\
& $7 / 3$ & 144.1 & 4.50 \\
& $8 / 2$ & 125.5 & 1.71 \\
$1 / 15(\mathrm{LR}=15)$ & $9 / 1$ & $\mathrm{NE}$ & 6.03 \\
& $5 / 5$ & 72.2 & $\mathrm{NE}$ \\
& $7 / 3$ & 91.1 & 6.93 \\
& $8 / 2$ & 85.5 & 2.95 \\
& $9 / 1$ & $\mathrm{NE}$ & $\mathrm{NE}$
\end{tabular}

m-Glc, methyl- $\alpha$-D-glucoside; CL, $\varepsilon$-caprolactone; LACD, L-lactide; $\mathrm{LR}$, liquid ratio; NE, not evaluated 
Thermal properties

\section{Polyols synthesized from $m-G l c / C L$ and m-Glc/LACD systems}

A series of DSC thermograms for polyols prepared by ringopening polymerization of CL in the presence of $\mathrm{m}-\mathrm{Glc}$ and $\mathrm{SnEht}_{2}$ are shown in Fig. 6. In the DSC data, $T_{\mathrm{g}}$ can be detected for all the samples and tends to decrease with an increase in the LR value. No melting peak was observed for two polyols synthesized with LRs of 2 and 5. In the case of LR of 5, the aggregation of the introduced CL side chains was likely so that the product assumed a solid-like state at room temperature. However, the DSC result indicated that the material did not reach crystallization. For development of the crystalline structure of PCL, a higher molecular weight of the product would be required (see Fig. 4).

When the LR reached 10 and over, the DSC thermograms of the synthesized polyols gave a clear melting signal above the respective $T_{\mathrm{g}}$ values; however, it was essentially composed of double peaks, as seen in Fig. 4. The maximum

(a)

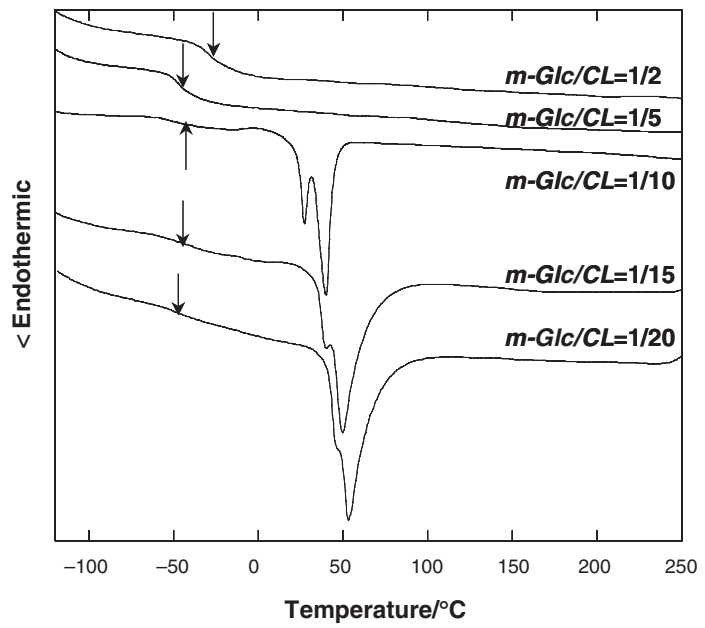

(b)

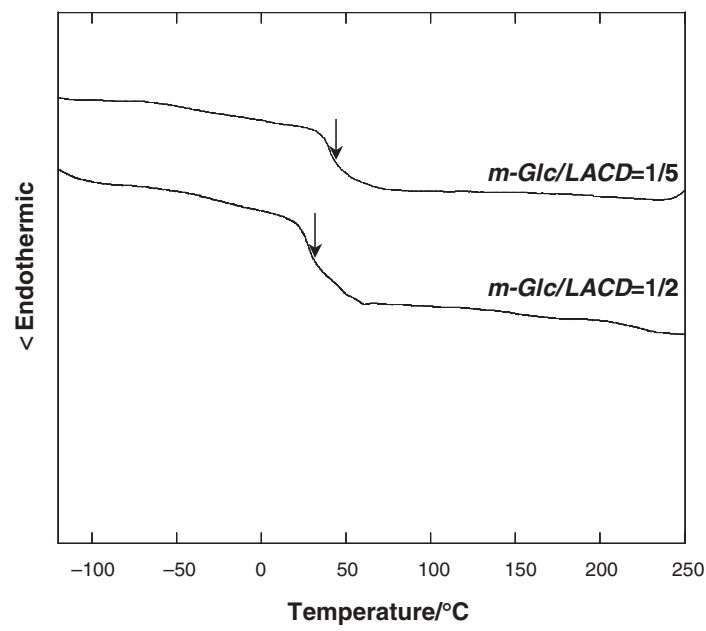

Fig. 6a,b. Differential scanning calorimetry (DSC) thermograms (2nd run) of polyols synthesized under conditions of $150^{\circ} \mathrm{C} / 150 \mathrm{~min}$. Arrows indicate $T_{\mathrm{g}}$ values position (the top of the larger peak) of the dual endotherms shifted to higher temperature with increasing LR, to come close to a $T_{\mathrm{m}}$ value $\left(\mathrm{ca} .60^{\circ} \mathrm{C}\right.$ ) of plain PCL of high molecular weight, which usually exhibits a single, sharper endotherm centered at the $T_{\mathrm{m}}$ value. $^{9-11}$ The smaller peak or shoulder observed before the emergence of the maximum endotherm in the heating process may be ascribed to the fusion of imperfect crystalline parts. The attachment of PCL side chains to the $\mathrm{m}-\mathrm{Glc}$ core can be regarded as being responsible for the inhibition of more complete crystallization. The relative strength of the shoulder decreased with an increase in the LR value, i.e., with an increase in the molecular weight of the PCL side chains (see Fig. 6). The behavior may be, more directly, attributable to the increase in the average degree of polymerization of the PCL side chains with LR. It follows that the increase results in the reduction of the number of PCL chain ends per unit volume of the polyol sample. This should become favorable for unrestrained growth of the crystalline structure.

DSC thermograms for two polyols prepared by the ringopening polymerization of LACD in the presence of m-Glc and $\mathrm{SnEht}_{2}$ are shown in Fig. 4. Taking account of $T_{\mathrm{g}} \fallingdotseq 59^{\circ} \mathrm{C}$ and $T_{\mathrm{m}} \fallingdotseq 178^{\circ} \mathrm{C}$ for conventional polylactide (PLA), ${ }^{12}$ it can be presumed that the side chains of the polyol samples have a still lower degree of polymerization of lactides and no regular association among them takes place, although the polyols assume a solid state at room temperature. The $T_{\mathrm{g}}$ values were both less than $50^{\circ} \mathrm{C}$, and that of the sample prepared with a larger LR was higher. No melting peak appeared in the range of the measurements.

\section{Polyols synthesized from m-Glc/(CL + LACD) system}

A series of DSC thermograms for polyols prepared by the ring-opening copolymerization of CL and LACD in the presence of $\mathrm{m}-$ Glc and $\mathrm{SnEht}_{2}$ are shown in Fig. 7. Figure 7a contains data for samples synthesized at a fixed LR of 5 and with different reactant molar ratios of CL:LACD ranging from $5: 5$ to $8: 2$, together with two control results. It can be seen in Fig. 7 that there is a successive increase in $T_{\mathrm{g}}$ from a value for the PCL-introduced $\mathrm{m}$-Glc (a control) to that for the PLA-introduced one (the other control) with the increase proportional to the LACD component. The thermograms show no melting peak, including the cases for the two controls. This indicates that there is no chance to form a crystalline structure among the introduced side chains, chiefly due to the small LR value of 5 .

A similar systematic dependence of $T_{\mathrm{g}}$ on the monomer composition was observed even when the LR value went up from 5 to 10 and 15, as is demonstrated in Fig. 7b,c. Concerning the formation of crystalline structure among the introduced side-chains, it is found unambiguously from these data that the copolymerization played a large role in preventing ordering. As can be seen from Fig. 7b,c, the products synthesized using CL:LACD ratios of 5:5-8:2 gave no melting peak, in contrast to the result for the solely PCL-introduced products of with LR values of 10 and 15 (see Fig. 6a). This means that the presence of at least $20 \mathrm{~mol} \%$ of LACD prevents the crystallization of the side 
(a)

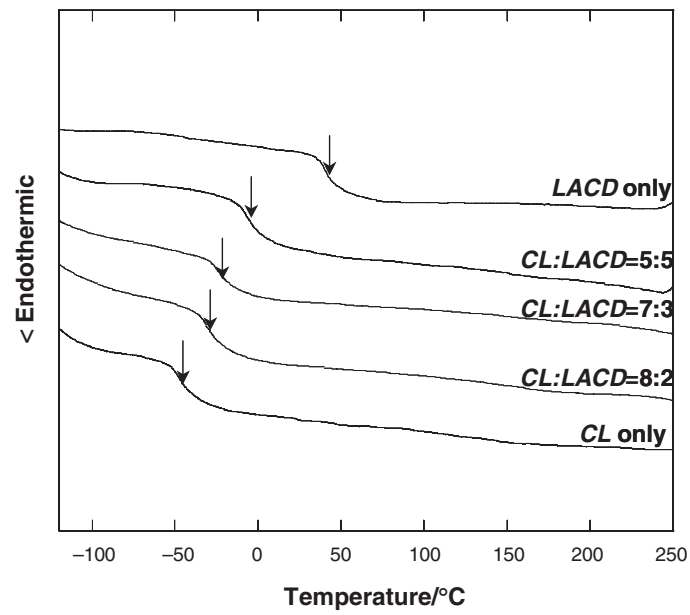

(b)

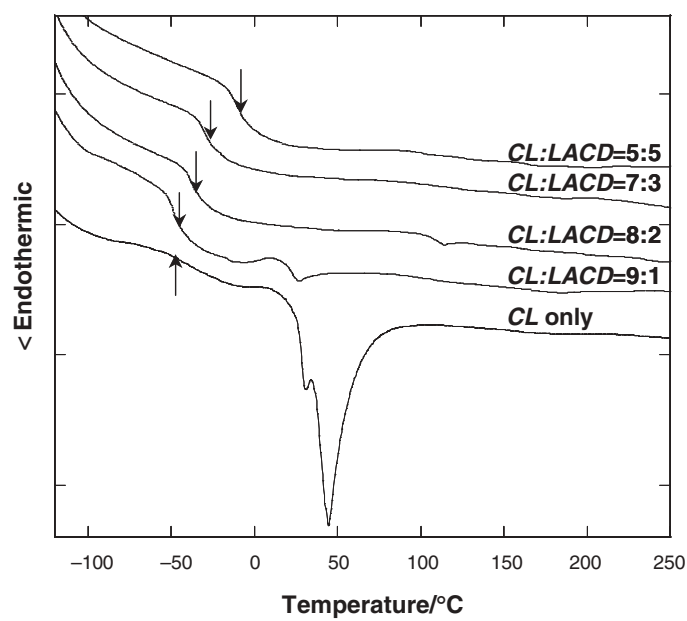

(c)

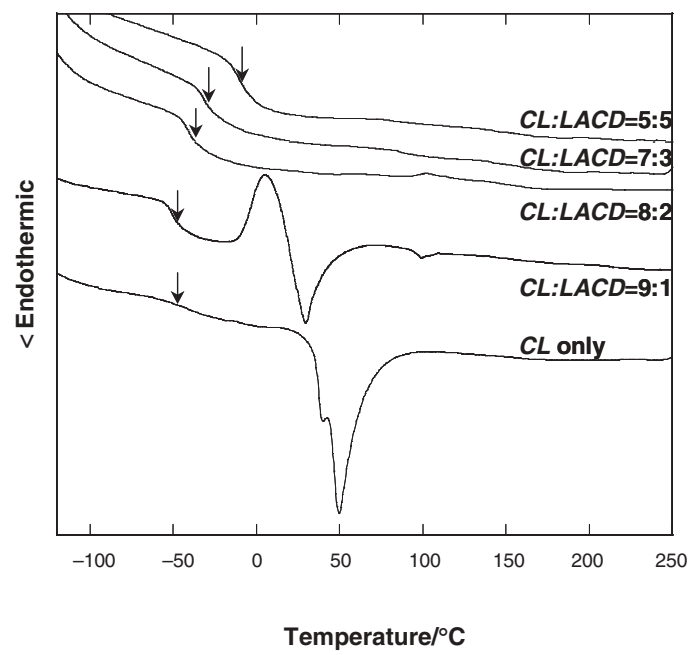

Fig. 7a-c. DSC thermograms for polyols synthesized under conditions of $150^{\circ} \mathrm{C} / 150 \mathrm{~min}$. (a) $\mathrm{LR}=5$; (b) $\mathrm{LR}=10$; (c) $\mathrm{LR}=15$. Arrows indicate $T_{\mathrm{g}}$ values

chains of polyols. When the molar content of CL reached $90 \%$ in the monomer composition, however, a sequence of signals of the crystallization and melting of PCL became recognizable for the polyol products synthesized with LR
Table 2. Mechanical properties of polyurethane foams

\begin{tabular}{|c|c|c|c|c|}
\hline Sample code & $\# 1$ & $\# 2$ & $\# 3$ & $\# 4$ \\
\hline Polyol (g) & $60^{\mathrm{a}}$ & $70^{\mathrm{b}}$ & $61^{\mathrm{c}}$ & $61^{\mathrm{d}}$ \\
\hline $\mathrm{H}_{2} \mathrm{O}(\mathrm{g})$ & 3.6 & 4.2 & 3.0 & 3.6 \\
\hline Surfactant (g) & 2.4 & 2.8 & 3.5 & 3.6 \\
\hline Catalyst (g) & 0.72 & 0.50 & 0.70 & 0.6 \\
\hline $\operatorname{MDI}(\mathrm{g})$ & 175 & 133 & 96.0 & 103 \\
\hline Density $\left(\mathrm{kg} / \mathrm{m}^{3}\right)$ & 56.9 & 29.1 & 36.7 & 32.7 \\
\hline Compre & (//) 245 & 160 & 268 & 149 \\
\hline relative def & $(\perp) \quad 92.8$ & 110 & 109 & 105 \\
\hline Compressive modulus of & $\begin{array}{ll}(/ /) & 7.58 \\
& 1.59\end{array}$ & $\begin{array}{l}5.01 \\
275\end{array}$ & $\begin{array}{c}11.2 \\
2.22\end{array}$ & $\begin{array}{l}6.58 \\
2.48\end{array}$ \\
\hline
\end{tabular}

SnEht $2: 0.33 \%$ / total (by weight); reaction temperature and time for synthesizing polyol: $150^{\circ} \mathrm{C}$ and $150 \mathrm{~min}$

//, Measured parallel to the foaming direction; $\perp$, measured perpendicular to the foaming direction

In-feed ratio (by weight):

${ }^{\mathrm{a}} \mathrm{m}-\mathrm{Glc} / \mathrm{CL}=1 / 4(\mathrm{LR}=4)$

${ }^{\mathrm{b}} \mathrm{m}-\mathrm{Glc} / \mathrm{CL}=1 / 3(\mathrm{LR}=3)$

${ }^{c} \mathrm{~m}-\mathrm{Glc} /(\mathrm{CL}+\mathrm{LACD})=1 / 5(\mathrm{LR}=5),(\mathrm{CL} / \mathrm{LACD}=80 / 20($ by mole $)$

${ }^{\mathrm{d}} \mathrm{m}-\mathrm{Glc} /(\mathrm{CL}+\mathrm{LACD})=1 / 5(\mathrm{LR}=5),(\mathrm{CL} / \mathrm{LACD}=70 / 30$ (by mole $)$

values of 10 and 15 . This trend became conspicuous with increasing LR. Actually, the use of a remarkably higher LR resulted in the formation of side chains of higher molecular weights, accelerating their crystallization and at the same time increasing the viscosity of the generated polyols, both of which reduce the practical performance of the products.

Mechanical properties of polyurethane foams

Polyurethane foams were prepared from four different polyols synthesized according to the formulations shown in Table 2. Data for the mechanical properties of the foams included densities of $29-57 \mathrm{~kg} / \mathrm{m}^{3}$, compression strengths at $10 \%$ strain of $150-270 \mathrm{kPa}$ (parallel to the foaming direction), and corresponding compression moduli of elasticity of $(5-11) \times 10^{3} \mathrm{kPa}$. These values all lie within a range indicating the potential practical use of the materials. The physical properties are expected to be further improved in future studies.

\section{Biodegradability}

Biodegradability was examined for two polyurethane foams, which were prepared from polyols synthesized by polymerization of CL in the presence of $\mathrm{m}-\mathrm{Glc}$ and $\mathrm{SnEht}_{2}$, using LRs of 2 and 5. Biodegradability was determined through measurements of the oxygen consumption in a closed system. The test samples were exposed to standard activated sludge. The degrees of degradation after 104 days of testing for the two samples were $41.4 \%$ (A, LR 2) and $15.6 \%$ (B, LR 5) (Table 3 and Fig. 8). Both samples were hydrophobic, and several days were required before the samples mixed with the suspensions. The test was discontinued after 104 days but further degradation can be expected, especially for the sample from the polyol synthesized with a LR of 2. According to the corresponding technical standard ISO 14851, 180 days of testing is permitted before attaining 
Table 3. Degrees of biodegradation of polyurethane foams estimated after 104 days

\begin{tabular}{lll}
\hline Samples & $\begin{array}{l}\text { Accumulated } \\
\mathrm{BOD} / \mathrm{mg}-\mathrm{O}_{2}\end{array}$ & $\begin{array}{l}\text { Degree of } \\
\text { biodegradation } / \%\end{array}$ \\
\hline $\mathrm{A}$ & 7.28 & 41.4 \\
$\mathrm{~B}$ & 2.85 & 15.6 \\
Control & Defined as 0 & \\
\hline
\end{tabular}

Degree of biodegradation $/ \%=(\mathrm{BOD} / \mathrm{ThOD}) \times 100 ; \mathrm{BOD}$ biochemical oxygen demand; ThOD, Theoretical oxygen demand (calculated based on elemental analysis for each foam); In-feed ratios for each polyol synthesis:

$A, \mathrm{~m}-\mathrm{Glc} / \mathrm{CL}=1 / 2(\mathrm{LR}=2) ; B, \mathrm{~m}-\mathrm{Glc} / \mathrm{CL}=1 / 5(\mathrm{LR}=5)($ by weight $)$

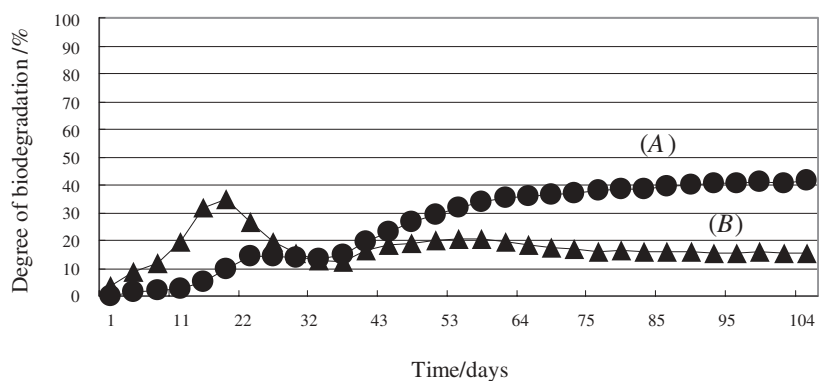

Fig. 8. Biodegradation profiles for polyurethane foams. In-feed ratios for each polyol synthesis: $\mathrm{A}, \mathrm{m}-\mathrm{Glc} / \mathrm{CL}=1 / 2(\mathrm{LR}=2) ; \mathrm{B}, \mathrm{m}-\mathrm{Glc} / \mathrm{CL}$ $=1 / 5(\mathrm{LR}=5)($ by weight $)$

plateau in the degree of biodegradation and if 60\% degradation is achieved up to that period, the sample is recognized as a biodegradable material. In that sense, the sample from the polyol synthesized with a LR of 2 may attain a significant level of biodegradability, although its structure was a three dimensionally cross-linked network.

The above-mentioned result demonstrates that biodegradability decreases with an increase in the molecular weight of polyols, i.e., in the quantity of CL introduced to $\mathrm{m}-\mathrm{Glc}$. This is considered to be due to the fact that regular aggregation of PCL side chains is strengthened with an increase in their length, making the attack by microorganisms difficult.

\section{Conclusions}

It is evident that $\mathrm{m}-\mathrm{Glc}$ provides viable polyols with $\mathrm{CL}$

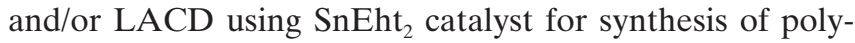
urethanes. For the polymerization in which only CL was employed as the ester monomer reagent, the products exhibited the following thermal behavior: the glass transition point $\left(T_{\mathrm{g}}\right)$ shifted to higher temperatures with a decrease in the reactant liquid ratio (LR) of the monomer to $\mathrm{m}-\mathrm{Glc}$, and the crystallization of PCL chains became noticeable with increasing LR. The resulting polyols were liable to have a high viscosity or to assume a solid state at room temperature. This is undesirable for a polyol material to be used as a component of polyurethane. To overcome this drawback, a ring-opening polymerization was conducted using a mixture of CL and LACD and it was found that occurrence of crystallization of the copolymer chains was considerably hindered. The copolymerization of $\mathrm{CL} /$ LACD, with CL:LACD ratios of $7: 3$ and $8: 2$, yielded a liquid product showing low viscosity in an acceptable LR range of monomer/m-Glc.

Polyurethane foams were prepared by using the polyols synthesized in combination with other chemicals that are commercially available. The physical properties of the foams obtained were found to be suitable for practical use.

The biodegradation test was performed by measuring oxygen consumption in an activated sludge suspension as a closed system. A significant level of biodegradation was confirmed for a polyurethane foam derived from a polyol prepared from PCL and m-Glc. This result suggests that polyurethane foams prepared from polyols containing high proportions of $\mathrm{m}$-Glc may show a high degree of biodegradability.

\section{References}

1. Hirose S, Hatakeyama H, Sano T, Teruya K (1998) The methods of preparing polyols from molasses. Japan Patent Unexamined 323200

2. Zetterlund $\mathrm{P}$, Hirose S, Hatakeyama T, Hatakeyama $H$, Albertsson A-C (1997) Thermal and mechanical properties of polyurethanes derived from mono- and disaccharides. Polym Int 42:1-8

3. Miyata A, Yoshioka M, Nishio Y (2002) Synthesis of glucose-based liquid polyol and its application to polyurethane foams. Abstracts of the 52nd Annual Meeting of the Japan Wood Research Society, Gifu, 479

4. Yoshioka M, Miyata A, Nishio Y (2004) Preparation of liquid polyesterpolyols from glucose and its methyl derivative. J Wood Sci 50:504-510

5. Hoshino T, Yoda N (1966) Preparative methods of polymer chemistry. Tokyo Kagaku Dojin, Tokyo, pp 118-119

6. Kricheldorf HR, Kreiser-Saunders I, Boettcher C (1995) Polylactones: 31. Sn (II) octoate-initiated polymerization of Llactide: a mechanistic study. Polymer 36:1253-1259

7. Yoshioka M, Hagiwara N, Shiraishi N (1999) Thermoplasticization of cellulose acetates by grafting of cyclic esters. Cellulose 6:193212

8. Imai Y (1987) Polyurethane foam. Kobunshi Kankokai, Kyoto, p 20

9. Nishio Y, Manley RSJ (1990) Blends of cellulose with nylon 6 and poly ( $\varepsilon$-caprolactone) prepared by a solution-coagulation method. Polym Eng Sci 30:71-82

10. Nishio Y, Matsuda K, Miyashita Y, Kimura N, Suzuki H (1997) Blends of poly ( $\varepsilon$-caprolactone) with cellulose alkyl esters: effect of alkyl side chain length and degree of substitution on miscibility. Cellulose 4:131-145

11. Lang M, Wong RP, Chu C-C (2002) Synthesis and structural analysis of functionalized poly ( $\varepsilon$-caprolactone)-based three-arm star polymers. J Polym Sci, Part A: Polym Chem 40:1127-1141

12. Ajioka M, Enomoto K, Suzuki K, Yamaguchi A (1995) The basic properties of poly (lactic acid) produced by the direct condensation polymerization of lactic acid. J Environ Polym Degrad 3:225-234 